\title{
Bringing the Background to the Foreground in Nursing Education: Appreciating Nursing Students' Backgrounds in an End-Of-Life Care Simulation
}

\author{
Michelle L Allen ${ }^{1 *}$ \\ ${ }^{1}$ EdD, MSN, RN, CCRN, CNE, CHSE, Department of Nursing, Dominican University
}

Received: December 20, 2017; Accepted: January 20, 2018; Published: January 24, 2018

*Corresponding author: Professor Michelle L Allen, EdD, MSN, RN, CCRN, CNE, CHSE, Lecturer, Dominican University, 7900 Division Street, River Forest, IL, USA; Tel: 847-261-2485; E-mail: mallen@dom.edu

\begin{abstract}
Background: Multiple healthcare agencies, such as American Association of Colleges of Nursing (2016) and American Nurses Association (2017), requested that end-of-life care be incorporated in undergraduate nursing education. Simulation offers nursing educators the opportunity to offer safe, hands-on end-of-life care education. However, a gap in the literature exists when examining the impact of the simulator and nursing students' backgrounds on their stress.

Objective: To examine the relationship between patient-type simulator and undergraduate nursing students' previous professional role exposure to individuals in high crisis on psychological and physiological stress.
\end{abstract}

Methods: The quantitative, quasi-experimental study involved analysis of physiological and psychological stress in 159 participants observing or actively providing care to a simulated end-of-life care patient. Further analysis involved assessing the impact of previous professional background exposure to individuals in high crises on stress levels. Psychological stress was analyzed through percentage changes in the State Trait Anxiety Inventory (STAI), Form Y-1; physiological stress was analyzed through percentage changes in heart rate, systolic and diastolic blood pressure.

Results: Overall greater psychological stress occurred when patient care involved the high-fidelity mannequin. Whereas, the greatest change in heart rate occurred in those with no professional exposure to individuals in high crises when care involved the standardized patient. However, the greatest percentage change in blood pressure occurred in those engaged with the care of the standardized patient.

Conclusion: There was a relationship between undergraduate nursing student stress, choice of patient simulator, and previous professional role exposure to individuals in high crises.

Keywords: End-of-life Care; Simulation; Nursing Education; Stress

\section{Introduction}

New graduate nurses have expressed dissatisfaction with their educational preparation on caring for death and dying patients [1]. The lack of preparation is a contributing factor to new graduate nursing stress $[2,3]$. At the same time, multiple organizations, such as the American Nurses' Association [ANA] and the American Association of Colleges of Nursing [AACN], have requested palliative care and end-of-life care be incorporated in undergraduate nursing education. The NCLEX-RN test blueprint, set into effect for April 2016 to through March of 2019, incorporates end-of-life care [4-6].

\section{Simulation as a Driving Force for End-Of-Life Care Preparation}

Simulation serves as an ideal facilitator of learning--connecting end-of-life care didactic content and clinical exposure. Rasmansama Vekataslu, Kellher and Hua Shao [2015] found that nursing students preferred learning about end-of-life care in the simulation laboratory as opposed to the traditional classroom [7]. Simulation serves as a teaching-learning strategy that provides learners the ability to practice in a safe, realistic healthcare environment tending to the needs of a simulated patient [8]. Selecting a student-centered simulated patient is dependent upon the needs of the learners. The simulated patient, called a simulator, in nursing and healthcare education has frequently been a highfidelity mannequin or standardized patient [9].The high-fidelity mannequin is a high-technology mannequin that mimics the "real-life" functions of a human patient. Standardized patients are live actors or actresses that serve as simulated patients [10]. Between, the two, the use of the high-fidelity mannequin has been more common in nursing education practices.

However, end-of-life care simulation research that has examined nursing student stress is scant $[9,11-13]$. Analyzing the relationship between nursing student stress, simulators, and their working backgrounds is lacking in the literature.

\section{Nursing Students' Background Are Left In The Background}

Creating a student-centered curriculum means identifying the needs of the learners in a way that will engage them in the content [14]. According to the Funds of Knowledge theory, each learner brings forth a background filled with various life experiences, knowledge, and skills that can impact their learning [15]. The background of individuals includes various exposures to individuals or experiences while at work. Recent trends have 
demonstrated an increase in nursing students working while they attend nursing school $[16,17]$. Understanding nursing students' working backgrounds and how that impacts their stress will provide nursing educators with the ability to select a simulator based upon their students' needs.

\section{Method}

The purpose of this quasi-experimental study was to analyze the impact previous professional exposures to individuals in high crises and simulators had on undergraduate nursing student stress. Participants cared for either the high-fidelity mannequin or standardized patient simulator. Stress was examined through psychological and physiological changes. Psychological changes were assessed through changes in the State and Trait Anxiety Inventory [STAI] Form Y-1. The STAI Form Y-1 was selected due to excellent reliability with a test-re-test median alpha coefficient of 0.92 and construct validity was assessed through factor analysis [18]. Physiological changes were assessed through changes in the participants' heart rate, systolic and diastolic blood pressures. These measurements assessed for changes in the "fight-or-flight" stress response of the autonomic nervous system.

\section{Participants}

Participants were invited to participate through email and a face-to-face in-class discussion about the study. From there, the participants signed up through an anonymous, online scheduling tool to participate in the simulation. Each participant signed up to be in a group comprised of five to eight participants.

Stratified random sampling, through randomization software, was used to determine simulated patient-type and learner-type. Patient-type was either a high-fidelity mannequin or standardized patient. Learner-type was either an active or observing learner where the active learner provided care to the dying simulated patient and the observing learner watched the care being provided during the simulation.

The a priori calculations identified that a minimum participant sample size was 120 . This study had 159 total participants, involved with or observing the care of the high-fidelity mannequin [ $\mathrm{n}=79]$ or standardized patient $[\mathrm{n}=80$ ] [19]. Prior to the simulation, participants were given a demographics form. The demographics form requested participants provide the number of professional roles in which they were exposed to individuals in high crises. Examples of such professional roles included: veteran of armed forces, patient care technician, emergency care technician, police officer, certified nursing assistant, etc.

\section{Procedure}

When the nursing students arrived to the simulation laboratory, they were provided with informed consent, including being made aware of the on-site services of the wellness and counseling centers. The nursing students were asked if they agreed to continue with participation and when agreed, they were given a packet of papers. The packet of papers consisted of a demographics form, table to document learner-type and patienttype as well as pre and post-simulation heart and blood pressure, pre-simulation STAI Form Y-2, and pre and post-simulation STAI Form Y-1.

After the participants filled out the demographics form and randomization of simulated patient-type and learner-type occurred, participants filled out the STAI Form Y-1 and Y-2. For this analysis, State Anxiety, Y-1 was used. From there, participants partnered-up, took each other's heart rate and obtained blood pressure per automated blood pressure cuff. Each participant documented their own heart and blood pressure findings in the table.

At that point, participants were given report on the 24 yearold, simulated patient dying of hepatic cancer and complications of Hepatitis C. Participants cared for or observed the care of either the high-fidelity mannequin or standardized patient. The simulation lasted 30 minutes. The patient required palliative care, did not have family at the bedside, and participants were required to provide post-mortem care including placing the simulated patient in a body bag. Extra care for the standardized patient occurred as participants placed a static mannequin in the body bag when post-mortem care was completed. The simulation was concluded once the body bag was zipped up and participants left the room.

Upon completion of the simulation, all participants obtained heart rate, systolic and diastolic blood pressure, and filled out the STAI Form Y-1 again. To compensate the participants for their participation, each was given a $\$ 10$ coffee shop gift card. After that, 30 minutes of debriefing occurred including discussion about nursing self-care coping strategies that can be utilized when providing palliative and end-of-life care to patients.

\section{Results}

Data was entered into SPSS 21.0 for analysis. Psychological measurement examined the percentage change in STAI, Form Y-1 scores; whereas physiological measurements examined the percentage change in heart rate, systolic and diastolic blood pressures. The analysis of findings incorporated the use of independent t-tests and two-way ANOVAs for both psychological and physiological stress measurements. The number of professional roles in which the individual was exposed to individuals in high crises were separated into categories of: none [no professional roles], low [one professional role], and medium [two professional roles] [Table 1].

Table 1: Number of Professional Roles Where Student Had Exposure to Individuals in High Crisis Situations

\begin{tabular}{|c|c|c|}
\hline $\begin{array}{c}\text { Number of Professional } \\
\text { Fields }\end{array}$ & $\mathbf{n}$ & $\mathbf{\%}$ \\
\hline None & 88 & 55.3 \\
\hline Low & 58 & 36.5 \\
\hline Medium & 13 & 8.2 \\
\hline Total & 159 & 100 \\
\hline
\end{tabular}




\section{Patient-Type on Stress}

The between-group differences analyzed the variations in patient-type on physiological and psychological stress [see Table 2]. While there were no between-group differences on patient type for physiological stress, psychological stress based on simulated-patient type identified a statistically significant difference. The high-fidelity mannequin $[M=29.55, S D=38.41]$ yielded greater percentage changes in psychological stress than the use of the standardized patient $[\mathrm{M}=18.00, \mathrm{SD}=28.19$; [ $\mathrm{t}(157)$ $=2.16, \mathrm{p}=0.03]$. The magnitude of the differences in the means was small [Cohen's d=0.34] [Table 2] [19].

Table 2: Interaction of Patient Type and Stress

\begin{tabular}{|c|c|c|c|} 
Patient-types & \multicolumn{2}{|c|}{ Physiological Stress (p value) } & $\begin{array}{c}\text { Psychological } \\
\text { Stress (p value) }\end{array}$ \\
\hline $\begin{array}{c}\text { High-Fidelity } \\
\text { Mannequin } \\
\text { (n=79) and }\end{array}$ & Heart Rate & $\begin{array}{c}\mathrm{t}(157)-0.23, \\
\mathrm{p}=0.82\end{array}$ & \\
\cline { 2 - 3 } $\begin{array}{c}\text { Standardized } \\
\text { Patient (n=80) }\end{array}$ & $\begin{array}{c}\text { Pressure } \\
\text { Diastolic Blood } \\
\text { Pressure }\end{array}$ & $\begin{array}{c}\mathrm{t}(157)=0.96, \\
\mathrm{p}=0.34\end{array}$ & $\mathrm{t}(157)=2.16, \mathrm{p}=0.03$ \\
& $\mathrm{t}(15)=0.21$ & \\
\hline
\end{tabular}

\section{Number of Professional Roles Participants Were} Exposed To Individuals In High Crisis

Heart Rate: Physiological stress, as measured by percentage change in heart rate yielded statistically significant results. There was a statistically significant interaction effect between patienttype and number of professional roles on the percentage change in heart rate $[F(2,153)=4.30, p=0.02]$; a small effect size was identified $[\eta 2=0.05]$. The fewer amounts of professional roles exposed to individuals in high crises, the greater the percentage change in heart rate when care involved the standardized patient [Table 3] [19].

Table 3: Percentage Change in Heart Rate

\begin{tabular}{|c|c|c|c|}
\hline Patient Type & $\begin{array}{c}\text { Number of } \\
\text { Professional Roles } \\
\text { Exposed to Individuals } \\
\text { in High Crisis }\end{array}$ & M, SD & $\mathbf{N}$ \\
\hline \multirow{4}{*}{$\begin{array}{l}\text { High-Fidelity } \\
\text { Mannequin }\end{array}$} & None & $2.05,11.19$ & 40 \\
\hline & Low & $6.81,14.40$ & 32 \\
\hline & Medium & $4.07,9.50$ & 7 \\
\hline & Total & $4.15,12.53$ & 79 \\
\hline \multirow{4}{*}{$\begin{array}{c}\text { Standardized } \\
\text { Patient }\end{array}$} & None & $6.95,16.14$ & 48 \\
\hline & Low & $-1.18,9.47$ & 26 \\
\hline & Medium & $-1.61,12.76$ & 6 \\
\hline & Total & $3.67,14.50$ & 80 \\
\hline \multirow{4}{*}{ Total } & None & $4.72,14.25$ & 88 \\
\hline & Low & $3.23,12.97$ & 58 \\
\hline & Medium & $1.45,11.03$ & 12 \\
\hline & Total & $3.90,13.52$ & 159 \\
\hline
\end{tabular}

Blood Pressure: Analysis of blood pressure was performed for both systolic and diastolic blood pressures. Systolic blood pressure demonstrated statistically significant results for changes in physiological stress. There was a statistically significant interaction effect between patient-type and number of professional roles on the percentage change in systolic blood pressure $[F(2,153)=3.26, p=0.04]$ with a small effect size $[\eta 2=0.04]$. The greatest percentage change in systolic blood pressure occurred when care involved the standardized patient and the participants worked in two or more professions that exposed them to individuals in high crisis [Table 4] [19]. However, there was no statistical significance for the interaction between patient-type and number of professional roles on the percentage change in diastolic blood pressure $[\mathrm{F}(2,153)=0.012, \mathrm{p}=0.89]$ [Table 5] [19].

Table 4: Percentage Change in Systolic Blood Pressure

\begin{tabular}{|c|c|c|c|}
\hline Patient Type & $\begin{array}{c}\text { Number of } \\
\text { Professional } \\
\text { Roles Exposed to } \\
\text { Individuals in High } \\
\text { Crisis }\end{array}$ & M, SD & N \\
\hline \multirow{2}{*}{$\begin{array}{c}\text { High-Fidelity } \\
\text { Mannequin }\end{array}$} & None & $2.79,10.36$ & 40 \\
\cline { 2 - 4 } & Low & $3.80,16.73$ & 32 \\
\hline \multirow{2}{*}{ Standardized } & Total & $-2.93,8.75$ & 7 \\
\hline Patient & None & $2.69,13.19$ & 79 \\
\cline { 2 - 4 } & Ledium & $-0.66,8.84$ & 28 \\
\hline \multirow{2}{*}{ Total } & Total & $1.18,10.34$ & 26 \\
\hline & None & $0.86,10.86$ & 80 \\
\hline & Low & $2.62,14.65$ & 88 \\
\hline & Total & $1.17,12.07$ & 58 \\
\hline & & $3.78,16.61$ & 13 \\
\hline
\end{tabular}

Table 5: Percentage Change in Diastolic Blood Pressure

\begin{tabular}{|c|c|c|c|}
\hline Patient Type & $\begin{array}{c}\text { Number of } \\
\text { Professional } \\
\text { Roles Exposed to } \\
\text { Individuals in High } \\
\text { Crisis }\end{array}$ & M, SD & $\mathbf{N}$ \\
\hline \multirow{4}{*}{$\begin{array}{c}\text { High-Fidelity } \\
\text { Mannequin }\end{array}$} & None & $4.01,10.82$ & 40 \\
\hline & Low & $6.15,13.45$ & 32 \\
\hline & Medium & $1.39,3.44$ & 7 \\
\hline & Total & $4.64,11.55$ & 79 \\
\hline \multirow{4}{*}{$\begin{array}{l}\text { Standardized } \\
\text { Patient }\end{array}$} & None & $1.93,7.99$ & 48 \\
\hline & Low & $5.20,7.96$ & 26 \\
\hline & Medium & $-2.24,5.76$ & 6 \\
\hline & Total & $2.68,8.03$ & 80 \\
\hline \multirow{4}{*}{ Total } & None & $2.87,9.38$ & 88 \\
\hline & Low & $5.72,11.25$ & 15 \\
\hline & Medium & $-0.28,4.83$ & 13 \\
\hline & Total & $3.66,9.95$ & 159 \\
\hline
\end{tabular}


STAI: Psychological stress was measured by percentage change in the STAI, Form Y-1 scores. There was no statistical significance interaction effect between patient type and the amount of professional roles in which the participant was exposed to individuals in high crisis and patient-type on psychological stress $[\mathrm{F}(2,153)=0.17, \mathrm{p}=0.85]$ [Table 6] [19].

Table 6: Percentage Change in STAI, Form Y-1 Scores

\begin{tabular}{|c|c|c|c|}
\hline Patient Type & $\begin{array}{c}\text { Number of } \\
\text { Professional } \\
\text { Roles Exposed to } \\
\text { Individuals in High } \\
\text { Crisis }\end{array}$ & M, SD & $\mathbf{N}$ \\
\hline \multirow{4}{*}{$\begin{array}{l}\text { High-Fidelity } \\
\text { Mannequin }\end{array}$} & None & $30.45,37.84$ & 40 \\
\hline & Low & $31.07,42.28$ & 32 \\
\hline & Medium & $17.44,21.24$ & 7 \\
\hline & Total & $29.55,38.41$ & 79 \\
\hline \multirow{4}{*}{$\begin{array}{c}\text { Standardized } \\
\text { Patient }\end{array}$} & None & $18.98,31.07$ & 48 \\
\hline & Low & $16.85,24.80$ & 26 \\
\hline & Medium & $15.20,19.44$ & 6 \\
\hline & Total & $18.00,28.19$ & 80 \\
\hline \multirow{4}{*}{ Total } & None & $24.19,34.59$ & 88 \\
\hline & Low & $24.70,35.95$ & 15 \\
\hline & Medium & $16.41,19.61$ & 13 \\
\hline & Total & $23.74,34.05$ & 159 \\
\hline
\end{tabular}

\section{Limitations}

This study was conducted at one university in suburban Chicago, Illinois, United States. The simulated patient was a young, adult female patient, which was similar to a majority of the participants' ages and gender. The scope of this research did not take into account the combined impact of learnertype and previous professional exposure on psychological and physiological stress in undergraduate nursing students participating in an end-of-life care simulation. As such, further research is needed to identify the impact of serving as an active or observing learner with previous professional exposure to end-of-life care on psychological and physiological stress in undergraduate nursing students participating in an end-of-life care simulation. Recommendations for further research include: a multi-site study, assess differences in stress when care is being provided to different ages and genders, and the impact of debriefing on psychological and physiological stress when nursing students are involved in an end-of-life care simulation.

\section{Discussion}

Overall, the greater psychological stress for undergraduate nursing students occurred with the high-fidelity mannequin. However, when bringing the background to the foreground, the results demonstrated different findings. Specifically, the physiological stress measurements demonstrated opposing findings. The greatest physiological stress in terms of percentage change of heart rate occurred when care involved the standardized patient and in those who had not worked with individuals in high crises. In addition, the nursing students who worked in two professions that exposed them to individuals in high crises, the greatest physiological stress as measured by percentage change in systolic blood pressure occurred with the care of the standardized patient.

When the researcher observed students working with the standardized patient, the students appeared more anxious. Often times, the students, particularly without exposure to end-oflife care, appeared confused and laughed nervously with each other, including when calling the physician to pronounce the standardized patient as deceased. It is possible that this anxiety led to the increase in physiological stress with the standardized patient. In addition, it is possible that previous memories in the participants who worked in two professions with individuals in high crisis led to a greater fight-or-flight response. A future qualitative component would promote greater understanding regarding the specific stressors of each patient-type.

New graduate nurses have described feeling anxious, uncertainty, and stressed to the point of choosing to leave the profession within the first year of practice $[1,20,21]$. The nursing profession has pushed to incorporate palliative care in nursing education through programs, such as the End-of-Life Nursing Education Consortium [ELNEC] [5]. Given this and understanding the results of this study, incorporating end-of-life care education as a simulation is necessary. Going forward, when nursing faculty implement an end-of-life care simulation experience, this research can offer insight into selecting a student-centered patient simulator based upon their students' working backgrounds.

\section{Acknowledgements}

This research was not funded by any grants. I appreciate the support of my dissertation committee at Aurora University: Dr. Joan Fee, Dr. Jay Thomas, and Dr. Charlene Thomas.

\section{Declarations}

- Conflict of interest: NA

- Ethical approval: This research was IRB-approved.

- Clinical trial registration: Written consent has been obtained.

\section{References}

1. Kwekkeboom KL, Vahl C, Eland J. Companionship and education: A nursing student experience in palliative care. J Nurs Educ. 2005; 44(4): 169-176.

2. Gagnon J, Duggleby W. The provision of end-of-life care by medical-surgical nurses working in acute care: A literature review. Palliative \& Supportive Care. 2014; 12(5); 393-408; Doi: 10.1017/ S1478951513000965

3. Peters L, Cant R, Payne S, O’Connor M, McDermott F, Hood K, et al. (2013, January). How death anxiety impacts nurses' caring for patients at the end of life: A review of literature. Open Nurs J. 2013; 7:14-21; Doi: $10.2174 / 1874434601307010014$

4. American Nurses Association. Call for action: Nurses lead and transform palliative care. 2017. 
5. American Association of Colleges of Nursing. ELNEC fact sheet. 2016.

6. National Council of State Boards of Nursing. Test plans. 2017.

7. Venkatasalu MR, Kelleher M, Shao CH. Reported clinical outcomes of high-fidelity simulation versus classroom-based end-of-life care education. Int J Palliat Nurs. 2015; 21(4):179-86; Doi: 10.12968/ ijpn.2015.21.4.179

8. Smith-Stoner M. Using high-fidelity simulation to educate nursing students about end-of-life care. Nurs Educ Perspect. 2009; 30(2):115120

9. Cavaye J, Watts JH. An integrated literature review of death education in pre-registration nursing curricula: Key themes. International Journal of Palliative Care. 2014. Doi: 10.1155/2014/564619

10.Association of Standardized Patient Educators. Overview. 2015.

11.Carman MJ. Bundling the death and dying learning experience for prelicensure nursing students. Nurse Educ. 2014; 39(3):135-137. Doi 10.1097/NNE.0000000000000036

12.Josephsen J, Martz K. Faculty and student perceptions: And end-of-life nursing curriculum survey. Journal of Hospice \& Palliative Nursing. 2014; 16(8): 474-481. Doi: 10.1097/NJH.0000000000000098

13.Fluharty L, Hayes AS, Milgrom L, Malarney K, Smith D, Reklau MA, et al A multisite, multi-academic track evaluation of end-of-life simulation for nursing education. Clinical Simulation in Nursing. 2012; 8(4): e135-e143; Doi: 10.1016/j.ecns.2010.08.003
14.International Society for Technology in Education. Student-centered learning. 2017.

15.Moll LC, Amanti C, Neff D, Gonzalez N. Funds of knowledge for teaching: Using a qualitative approach to connect homes and classrooms. Theory into Practice. 1992; 31(2): 132-141.

16.Pulido-Martos M, Augusto-Landa JM, Lopez-Zafra E. Sources of stress in nursing students: A systematic review of quantitative studies. International Nursing Review. 2012; 59(1): 15-25; Doi: 10.1111/j.14667657.2011.00939.x

17.Reyes H, Hartin V, Loftin C, Davenport D, Carter V. The impact of employment on nursing students' academic performance. Nurse Educ. 2012; 37(5):218-221; Doi: 10.1097/NNE.0b013e318262abc9

18.Spielberger CD. State-trait anxiety inventory for adults manual [Manual]. 2015.

19.Allen M. Examining the relationship between nursing student stress and use of technology in an end-of-life simulation. Clinical Simulation in Nursing. 2017; 14: 21-28; Doi: 10.1016/j.ecns.2017.10.006

20.Gillan PC, Parameter G, Van der Riet PJ, Jeong S. The experience of end of life care simulation at a rural Australian university. Nurse Education Today. 2013; 33(11): 1435-1439. Doi: 10.1016/j.nedt.2012.11.015

21.MacKusik CI, Minick P. Why are nurses leaving? Findings from an initial qualitative study on nursing attrition. MEDSURG Nursing, 19(6): 335340 